\title{
QUBIT PORTRAIT OF THE PHOTON-NUMBER TOMOGRAM AND SEPARABILITY OF TWO-MODE LIGHT STATES
}

\author{
Sergey N. Filippov ${ }^{1}$ and Vladimir I. Man'ko² \\ 1 Moscow Institute of Physics and Technology (State University) \\ Institutskii per. 9, Dolgoprudnyi, Moscow Region 141700, Russia \\ 2 P. N. Lebedev Physical Institute, Russian Academy of Sciences \\ Leninskii Prospect 53, Moscow 119991, Russia \\ e-mail: filippovsn@gmail.com manko@sci.lebedev.ru
}

\begin{abstract}
In view of the photon-number tomograms of two-mode light states, using the qubitportrait method for studying the probability distributions with infinite outputs, the separability and entanglement detection of the states are studied. Examples of entangled Gaussian state and Schrödinger cat state are discussed.
\end{abstract}

Keywords: qubit portrait, photon-number tomogram, entanglement of light states

\section{Introduction}

The probability representation of quantum states with continuous variables was suggested in [1. According to this representation, any quantum states is associated with a fair probabilitydistribution function called symplectic tomogram (or tomographic-probability distribution). Information contained in the tomographic-probability distribution is the same that is in the density operator. The optical tomograms [2, 3] were used earlier as a technical tool to reconstruct the Wigner function [4] which was identified with the quantum state. The experiments to measure quantum states [5] are aimed at obtaining the Wigner function by means of measuring the homodyne quadrature component. Though this procedure implies the measurement of optical tomograms, the latter ones were not identified with the quantum states in these experiments. The optical tomogram is considered as intermediate technical information that gives the opportunity to "reconstruct" the state identified with the Wigner function.

An understanding of the fact that tomograms (optical or symplectic ones) are themselves a primary notion of quantum states was suggested in [1] (see also [6, 7, 8, 9]). The analogues treatment of quantum states of spin systems (qubits, qudits) as tomographic-probability distributions was proposed in [10, 11]. Thus, in the probability representation of quantum mechanics, which is completely equivalent to other representations like Schrödinger representation [12], Feynmann path integral representation [13], Moyal representation [14], etc., the quantum states are described by the standard probability distributions (state tomograms). Spin tomograms were used to discuss the separability and entanglement phenomena in [15, 16, 17]. The 
qubit-portrait method for studying entanglement of multiqudit systems was suggested in [18]. This method is similar to the integration method of symplectic tomograms applied in [19] (see also [20]), with both methods providing the analogs of two-qubit states.

There exist descriptions of the photon states in terms of the photon-number tomograms [21, 22, 23]. The photon-number tomogram is the probability distribution of a discrete variable $n=0,1,2, \ldots$. This distribution function also depends on an extra complex parameter and contains complete information on the quantum state. The photon-number tomograms for the Gaussian states were constructed in [24]. The aim of our work is to develop an analog of the qubit portrait method that was suggested for qudit states and apply this method to the photonnumber tomogram of multimode photon systems. This means that we consider a linear map of discrete probability distributions with infinite probability vectors onto probability distributions with finite probability vectors. The map, which is applied to the photon-number tomograms, is not a positive map (see, e.g., [25]). Nevertheless, it provides a possibility to detect the entanglement of multimode photon states. Positive maps were used to detect entanglement in [26, 27, 28] and nonpositive maps were used to detect entanglement, e.g., in [29, 30, 31, 32, 33].

In this paper we focus on two-mode nonclassical states of light, which are of great interest since they can have some particular features [34, 35]. Entanglement [12] of quantum states turned out to be a very important tool for secure quantum communication and quantum cryptography. A geometrical interpretation of entangled two-qudit states was proposed in [36]. In spite of the fact that detection of entanglement has undergone rapid development in the last few decades, they do not cover all the possibilities. The main goal of the present article is to introduce an alternative method that can reveal the entanglement of two-mode light states. There have been already made successful attempts to solve this problem [28, 37], for instance, by using the Bell-CHSH inequality [38, 39] within the framework of symplectic tomograms [1, 19, 40]. Here, we try to attack the problem with the help of the photon-number tomogram [21, 22, 23] which provides additional information on quantum correlations.

Since the entanglement of two qubit system has been sufficiently investigated, it looks feasible to detect the entanglement of the system involved by using a linear map of the photon-number tomogram with infinite outputs onto qubit tomogram (qubit portrait). The qubit-portrait method to study qudit states was introduced in [18] and developed in [15]. As far as there exist many ways to construct a qubit portrait, we are going to discuss some examples of them. The procedure above results in reducing the separability property of a two-mode light state to the Bell-CHSH inequality for two qubits. That inequality is fulfilled if the initial two-mode light state is separable. It naturally leads to a necessary condition of separability. Its violation indicates immediately that the state in question is entangled.

The paper is organized as follows.

In Sec. 2, we give a brief review of the photon-number tomogram and its generalization to the two-mode case. Here we also suggest a method of obtaining the joint probability distribution corresponding to a two-qubit tomogram. In Sec. 3, an example of Schrödinger cat state is studied in detail. In Sec. 4, we introduce the general approach to deal with the Gaussian states. A particular example of its application is given and another way of constructing the qubit portrait is demonstrated. In Sec. 5, conclusions and prospects are presented. 


\section{Photon-number tomogram and its qubit portrait}

The conventional photon-number tomogram for a one-mode light state given by the density matrix $\hat{\rho}$ reads [21, 22, 23]

$$
w(n, \alpha)=\operatorname{Tr}\left(\hat{\rho} \hat{D}^{\dagger}(\alpha)|n\rangle\langle n| \hat{D}(\alpha)\right)=\left\langle n\left|\hat{D}(\alpha) \hat{\rho} \hat{D}^{\dagger}(\alpha)\right| n\right\rangle,
$$

where $|n\rangle$ is an eigenstate of the operator $\hat{a}^{\dagger} \hat{a}$, with $\hat{a}^{\dagger}$ and $\hat{a}$ being the photon creation and annihilation operators, respectively, and $\hat{D}(\alpha)$ is a displacement operator, which depends on the complex number $\alpha$ as follows:

$$
\hat{D}(\alpha)=\mathrm{e}^{\alpha \hat{a}^{\dagger}-\alpha^{*} \hat{a}} .
$$

Thus, the photon-number tomogram is nothing else but the photon distribution function of the state $\hat{D}^{\dagger}(\alpha) \hat{\rho} \hat{D}(\alpha)$. In other words, it is the probability to find $n$ photons in the state with the amplitude shifted by a complex number $\alpha$. It is worth noting that once tomogram (1) is known, the state $\hat{\rho}$ can be reconstructed. (For Gaussian states the photon distributions were expressed in terms of Hermite polynomials of several variables in [41, 42]).

As far as the two-mode light state is concerned, the formula (11) changes slightly

$$
w\left(n_{1}, n_{2}, \alpha_{1}, \alpha_{2}\right)=\left\langle n_{1} n_{2}\left|\hat{D}\left(\alpha_{1}, \alpha_{2}\right) \hat{\rho} \hat{D}^{\dagger}\left(\alpha_{1}, \alpha_{2}\right)\right| n_{1} n_{2}\right\rangle,
$$

where $\left|n_{1} n_{2}\right\rangle=\left|n_{1}\right\rangle\left|n_{2}\right\rangle$ is the state with $n_{1}$ photons in the first mode and $n_{2}$ photons in the second mode, and $\hat{D}\left(\alpha_{1}, \alpha_{2}\right)=\hat{D}_{1}\left(\alpha_{1}\right) \hat{D}_{2}\left(\alpha_{2}\right)$. Here $\hat{D}_{i}\left(\alpha_{i}\right)$ denotes the displacement operator (2), where we replaced $\alpha \rightarrow \alpha_{i}, \hat{a}^{\dagger} \rightarrow \hat{a}_{i}^{\dagger}$, and $\hat{a} \rightarrow \hat{a}_{i}$, with $\hat{a}_{i}^{\dagger}$ and $\hat{a}_{i}$ being the photon creation and annihilation operators of the $i$ th mode, $i=1,2$. The operators $\hat{D}_{1}\left(\alpha_{1}\right)$ and $\hat{D}_{2}\left(\alpha_{2}\right)$ obviously commute.

The photon-number tomogram (1) is rather similar to the spin tomogram [10, 11] of a qudit (particle with spin $j$ ). The number of photons $n$ plays the role of the spin projection $m$ $(m=-j,-j+1, \ldots, j)$, while the complex number $\alpha$ is analogues to the unitary matrix of the corresponding rotation group. In contrast to the spin tomogram, the photon-number tomogram has infinite outputs, so there are infinite ways to construct a qubit portrait [18, 15] of such

a tomogram. Indeed, if one has the infinite probability-distribution vector with nonnegative components

$$
\vec{W}_{\infty}=\left(\begin{array}{c}
w(0, \alpha) \\
w(1, \alpha) \\
w(2, \alpha) \\
\cdots
\end{array}\right)
$$

where $\sum_{n=1}^{\infty} w(n, \alpha)=1$, a new probability-distribution vector $\vec{W}_{2}$ with two components can be constructed as follows

$$
\vec{W}_{2}=\left(\begin{array}{c}
\sum_{n \in A} w(n, \alpha) \\
\sum_{n \in\left(Z_{+} \backslash A\right)} w(n, \alpha)
\end{array}\right)
$$




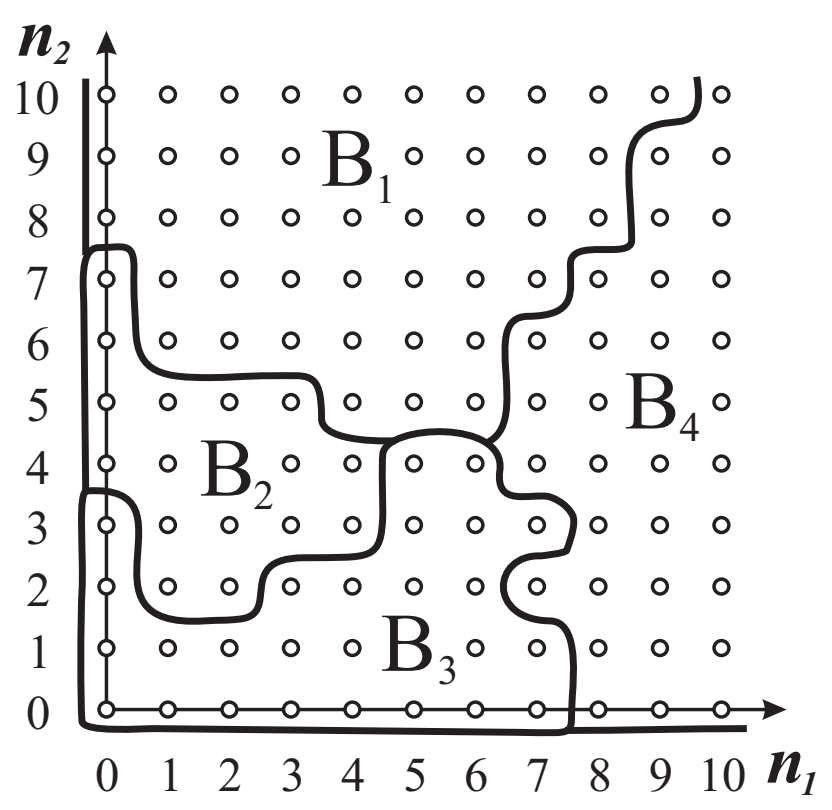

Figure 1: Partition of the set $Z_{+} \times Z_{+}$to subsets $B_{i}, i=1, \ldots, 4$, that enables one to construct the four-vector (7). Nevertheless, such a vector is not a probability distribution vector of two qubits because $B_{i}$ cannot be written in the form $A_{1}^{(i)} \times A_{2}^{(i)}$, where $A_{1}^{(i)}$ and $A_{2}^{(i)}$ are subsets of $Z_{+}$.

where $A$ is an arbitrary subset of the set of nonnegative integers $Z_{+} \equiv\{0,1,2, \ldots\}$. (We denote the set of nonnegative integers that do not belong to $A$ as $Z_{+} \backslash A$; from this it follows that $\left.A \cup\left(Z_{+} \backslash A\right)=Z_{+}, A \cap\left(Z_{+} \backslash A\right)=\emptyset\right)$.

Let us now consider the photon-number tomogram of the two-mode light.

Once the parameters $\alpha_{1}$ and $\alpha_{2}$ are given the function $w\left(n_{1}, n_{2}, \alpha_{1}, \alpha_{2}\right)$ can be treated as a table with an infinite number of both rows and columns

$$
\bar{W}_{\infty, \infty}=\left(\begin{array}{cccc}
w\left(0,0, \alpha_{1}, \alpha_{2}\right) & w\left(0,1, \alpha_{1}, \alpha_{2}\right) & w\left(0,2, \alpha_{1}, \alpha_{2}\right) & \ldots \\
w\left(1,0, \alpha_{1}, \alpha_{2}\right) & w\left(1,1, \alpha_{1}, \alpha_{2}\right) & w\left(1,2, \alpha_{1}, \alpha_{2}\right) & \ldots \\
w\left(2,0, \alpha_{1}, \alpha_{2}\right) & w\left(2,1, \alpha_{1}, \alpha_{2}\right) & w\left(2,2, \alpha_{1}, \alpha_{2}\right) & \ldots \\
\ldots & \ldots & \ldots & \ldots
\end{array}\right)
$$

with the sum $\sum_{n_{1}, n_{2}=0}^{\infty} w\left(n_{1}, n_{2}, \alpha_{1}, \alpha_{2}\right)$ being equal to unity. This distribution induces the fourdimensional probability distribution vector of the form

$$
\vec{W}_{4}^{\prime}=\left(\begin{array}{c}
\sum_{\left(n_{1}, n_{2}\right) \in B_{1}} w\left(n_{1}, n_{2}, \alpha_{1}, \alpha_{2}\right) \\
\sum_{\left(n_{1}, n_{2}\right) \in B_{2}} w\left(n_{1}, n_{2}, \alpha_{1}, \alpha_{2}\right) \\
\sum_{\left(n_{1}, n_{2}\right) \in B_{3}} w\left(n_{1}, n_{2}, \alpha_{1}, \alpha_{2}\right) \\
\sum_{\left(n_{1}, n_{2}\right) \in B_{4}} w\left(n_{1}, n_{2}, \alpha_{1}, \alpha_{2}\right)
\end{array}\right)
$$


where the sets $B_{i}(i=1, \ldots, 4)$ satisfy the conditions: $B_{i} \subset Z_{+} \times Z_{+}, \bigcup_{i=1}^{4} B_{i}=Z_{+} \times Z_{+}$, and $B_{i} \cap B_{j}=\emptyset$ if $i \neq j$. An example of separation of the set $Z_{+} \times Z_{+}$that meets all the above requirements is illustrated in Fig. 1.

The probability vector obtained can be interpreted readily as the spin tomogram of a particle with spin $j=3 / 2$, but it is hardly able to describe the two-qubit system. The matter is that the simply separable state of two qubits has the tomogram of a factorized form $w\left(m_{1}, m_{2}, \vec{N}_{1}, \vec{N}_{2}\right)=$ $w\left(m_{1}, \vec{N}_{1}\right) w\left(m_{2}, \vec{N}_{2}\right)$, where $m_{1}$ and $m_{2}$ are the spin projections of the first and second spin- $1 / 2$ particles to the axes $\vec{N}_{1}$ and $\vec{N}_{2}$, respectively. This feature underlies the Bell-CHSH inequality and is of a great importance. For the factorization above to be valid, it requires $B_{i}$ to be the direct product of subsets of $Z_{+}, i=1, \ldots, 4$. Consequently, once purposing to construct the qubit portrait, one should rewrite formula (7) in the form

$$
\vec{W}_{4}=\left(\begin{array}{rl} 
& \sum_{\left(n_{1}, n_{2}\right)} \in A_{1} \times A_{2} \\
& \sum_{A_{1} \times\left(Z_{+} \backslash A_{2}\right)} w\left(n_{1}, n_{2}, \alpha_{1}, \alpha_{2}\right) \\
\left(n_{1}, n_{2}\right) & \\
\left(n_{1}, n_{2}\right) & \left.\in \sum_{1}, \alpha_{2}\right) \\
\left(Z_{1}, n_{2}\right) & \sum_{\left(Z_{+} \backslash A_{1}\right) \times A_{2}} w\left(n_{1}, n_{2}, \alpha_{1}, \alpha_{2}\right) \\
& w\left(Z_{+} \backslash A_{2}\right)
\end{array}\right),
$$

where $A_{1}$ and $A_{2}$ are subsets of the set of nonnegative integers $Z_{+}$. If the state is simply separable, i.e.,

$$
w\left(n_{1}, n_{2}, \alpha_{1}, \alpha_{2}\right)=w_{1}\left(n_{1}, \alpha_{1}\right) w_{2}\left(n_{2}, \alpha_{2}\right),
$$

then vector (8) can be written in factorized form

$$
\left(\begin{array}{c}
\sum_{n_{1} \in A_{1}} w\left(n_{1}, \alpha_{1}\right) \\
\sum_{n_{1} \in\left(Z_{+} \backslash A_{1}\right)} w\left(n_{1}, \alpha_{1}\right)
\end{array}\right) \otimes\left(\begin{array}{c}
\sum_{n_{2} \in A_{2}} w\left(n_{2}, \alpha_{2}\right) \\
\sum_{n_{2} \in\left(Z_{+} \backslash A_{2}\right)} w\left(n_{2}, \alpha_{2}\right)
\end{array}\right),
$$

which corresponds to the probability-distribution vector of the simply separable two-qubit state. As far as separable states are concerned, the four-vector (8) becomes merely the convex sum of the vectors (10).

To draw an analogy with the probability-distribution vector of two qubits, we designate the components of the vector (8) as follows:

$$
\vec{W}_{4}=\left(\begin{array}{c}
w\left(+,+, \alpha_{1}, \alpha_{2}\right) \\
w\left(+,-, \alpha_{1}, \alpha_{2}\right) \\
w\left(-,+, \alpha_{1}, \alpha_{2}\right) \\
w\left(-,-, \alpha_{1}, \alpha_{2}\right)
\end{array}\right),
$$

with all the components being nonnegative and their sum equal to unity.

Comment. $\nabla$ The construction of the four-dimensional probability distribution vector from the matrix (6) can be presented in an elegant matrix form 1 . To start, we show how one can

\footnotetext{
${ }^{1}$ Such a map for finite probability vectors was used in the work of M. A. Man'ko, V. I. Man'ko, and R. V. Mendes (unpublished)
} 
obtain a probability-distribution vector $\vec{w}_{2}$ with two components with the help of a probabilitydistribution vector

$$
\vec{w}_{3}=\left(\begin{array}{c}
a \\
b \\
c
\end{array}\right)
$$

The general form of transformation $\vec{w}_{3} \rightarrow \vec{w}_{2}$ reads

$$
\vec{w}_{2}=\left(\begin{array}{c}
K \\
L
\end{array}\right)=\left(\begin{array}{c}
p_{1} a+p_{2} b+p_{3} c \\
1-\left(p_{1} a+p_{2} b+p_{3} c\right)
\end{array}\right)=\left(\begin{array}{ccc}
p_{1} & p_{2} & p_{3} \\
1-p_{1} & 1-p_{2} & 1-p_{3}
\end{array}\right)\left(\begin{array}{l}
a \\
b \\
c
\end{array}\right),
$$

where $p_{i} \geq 0$ and $\sum p_{i}=1$. Regarding $\vec{w}_{2}$ as a three-dimensional probability vector, one can write

$$
\left(\begin{array}{c}
\vec{w}_{2} \\
0
\end{array}\right)=\left(\begin{array}{c}
K \\
L \\
0
\end{array}\right)=\left(\begin{array}{ccc}
p_{1} & p_{2} & p_{3} \\
1-p_{1} & 1-p_{2} & 1-p_{3} \\
0 & 0 & 0
\end{array}\right)\left(\begin{array}{c}
a \\
b \\
c
\end{array}\right)=M \vec{w}_{3}
$$

where $M$ is a stochastic matrix with no more than two nonzero rows. A generalization of this method to higher dimensions is obvious, and one has

$$
\left(\begin{array}{c}
\vec{w}_{2} \\
0 \\
\cdots \\
0
\end{array}\right)=M_{\infty} \vec{w}_{\infty}
$$

To deal with the probability-distribution matrices, one can use their vector representation [18] or, equivalently, apply the technic above separately to the rows and columns. In the case of matrix (6) , we have

$$
\left(\begin{array}{ccccc}
w_{++} & w_{+-} & 0 & \ldots & 0 \\
w_{-+} & w_{--} & 0 & \ldots & 0 \\
0 & 0 & 0 & \ldots & 0 \\
\ldots & \ldots & \ldots & \ldots & \ldots \\
0 & 0 & 0 & \ldots & 0
\end{array}\right)=M_{\infty}^{(1)} \bar{W}_{\infty, \infty} M_{\infty}^{(2)}{ }^{T}
$$

where the block $\left(\begin{array}{cc}w_{++} & w_{+-} \\ w_{-+} & w_{--}\end{array}\right)$provides a required qubit portrait. This completes the Comment. $\triangle$

Since the four-vector (11) is merely a vector-function of parameters $\alpha_{1}$ and $\alpha_{2}$, we can introduce new complex variables $\beta_{1}$ and $\beta_{2}$ and construct the stochastic matrix of the form

$$
M\left(\alpha_{1}, \alpha_{2}, \beta_{1}, \beta_{2}\right)=\left(\begin{array}{llll}
w\left(+,+, \alpha_{1}, \alpha_{2}\right) & w\left(+,+, \alpha_{1}, \beta_{2}\right) & w\left(+,+, \beta_{1}, \alpha_{2}\right) & w\left(+,+, \beta_{1}, \beta_{2}\right) \\
w\left(+,-, \alpha_{1}, \alpha_{2}\right) & w\left(+,-, \alpha_{1}, \beta_{2}\right) & w\left(+,-, \beta_{1}, \alpha_{2}\right) & w\left(+,-, \beta_{1}, \beta_{2}\right) \\
w\left(-,+, \alpha_{1}, \alpha_{2}\right) & w\left(-,+, \alpha_{1}, \beta_{2}\right) & w\left(-,+, \beta_{1}, \alpha_{2}\right) & w\left(-,+, \beta_{1}, \beta_{2}\right) \\
w\left(-,-, \alpha_{1}, \alpha_{2}\right) & w\left(-,-, \alpha_{1}, \beta_{2}\right) & w\left(-,-, \beta_{1}, \alpha_{2}\right) & w\left(-,-, \beta_{1}, \beta_{2}\right)
\end{array}\right) .
$$


The matrix (17) is obtained just in the same way as its analog for a two-qubit system [43]. For this reason it exhibits the same properties. The most essential point is the following. If the initial state of two-mode light is separable then, the Bell-CHSH inequality is fulfilled for certain. That implies that the inequality

$$
\left|\operatorname{Tr}\left(M\left(\alpha_{1}, \alpha_{2}, \beta_{1}, \beta_{2}\right) I\right)\right| \leq 2
$$

holds true, where the matrix $I$ is given by the formula

$$
I=\left(\begin{array}{cccc}
1 & -1 & -1 & 1 \\
1 & -1 & -1 & 1 \\
1 & -1 & -1 & 1 \\
-1 & 1 & 1 & -1
\end{array}\right)
$$

If there exist complex numbers $\alpha_{1}, \alpha_{2}, \beta_{1}, \beta_{2}$ such that the inequality (18) is violated, the two-mode light state is entangled. Consequently, fulfilling the Bell-CHSH inequality is a necessary condition of separability of the two-mode light state.

\section{Detecting entanglement of the Schrödinger cat state}

To demonstrate the application of the technique described above, we consider the state that is entangled by construction. (One cannot help mentioning that the entanglement of the Schrödinger cat states was detected by means of symplectic tomograms in [20]). Here we investigate the Schrödinger cat state of the form [44, 45]

$$
|\psi\rangle=N\left(\gamma_{1}, \gamma_{2}\right)\left(\left|\gamma_{1}, \gamma_{2}\right\rangle+\left|-\gamma_{1},-\gamma_{2}\right\rangle\right),
$$

where $\left|\gamma_{1}, \gamma_{2}\right\rangle=\left|\gamma_{1}\right\rangle\left|\gamma_{2}\right\rangle$, states $\left|\gamma_{i}\right\rangle$ are eigenstates of the photon annihilation operators, i.e., $\hat{a}_{i}\left|\gamma_{i}\right\rangle=\gamma_{i}\left|\gamma_{i}\right\rangle, i=1,2$ (coherent states of the first and the second modes, respectively). The factor $N\left(\gamma_{1}, \gamma_{2}\right)$ can be found by employing the normalization requirement $\langle\psi \mid \psi\rangle=1$. Indeed, since for arbitrary coherent states $|\gamma\rangle$ and $|\delta\rangle$ the scalar product $\langle\gamma \mid \delta\rangle$ can be written in the form [46, 47]

$$
\langle\gamma \mid \delta\rangle=\exp \left\{-\frac{1}{2}|\gamma|^{2}-\frac{1}{2}|\delta|^{2}+\gamma^{*} \delta\right\},
$$

it is of no difficulty to calculate

$$
\begin{aligned}
\langle\psi \mid \psi\rangle & =N^{2}\left(\gamma_{1}, \gamma_{2}\right)\left(\left\langle\gamma_{1}, \gamma_{2} \mid \gamma_{1}, \gamma_{2}\right\rangle+2 \operatorname{Re}\left(\left\langle\gamma_{1}, \gamma_{2} \mid-\gamma_{1},-\gamma_{2}\right\rangle\right)+\left\langle-\gamma_{1},-\gamma_{2} \mid-\gamma_{1},-\gamma_{2}\right\rangle\right) \\
& =2 N^{2}\left(\gamma_{1}, \gamma_{2}\right)\left(1+\mathrm{e}^{-2\left|\gamma_{1}\right|^{2}-2\left|\gamma_{2}\right|^{2}}\right)=4 N^{2}\left(\gamma_{1}, \gamma_{2}\right) \mathrm{e}^{-\left(\left|\gamma_{1}\right|^{2}+\left|\gamma_{2}\right|^{2}\right)} \cosh \left(\left|\gamma_{1}\right|^{2}+\left|\gamma_{2}\right|^{2}\right) .
\end{aligned}
$$

Therefore,

$$
N\left(\gamma_{1}, \gamma_{2}\right)=\frac{\exp \left\{\frac{1}{2}\left(\left|\gamma_{1}\right|^{2}+\left|\gamma_{2}\right|^{2}\right)\right\}}{2\left\{\cosh \left(\left|\gamma_{1}\right|^{2}+\left|\gamma_{2}\right|^{2}\right)\right\}^{1 / 2}}
$$


In case of pure quantum state, i.e. $\hat{\rho}=|\psi\rangle\langle\psi|$, the formula (3) takes a more convenient form

$$
w_{\psi}\left(n_{1}, n_{2}, \alpha_{1}, \alpha_{2}\right)=\left|\left\langle n_{1} n_{2}\left|\hat{D}\left(\alpha_{1}, \alpha_{2}\right)\right| \psi\right\rangle\right|^{2}
$$

To get the explicit expression of the photon-number tomogram in our particular case, we recall that $|\gamma\rangle=\hat{D}(\gamma)|0\rangle$ and $\hat{D}(\alpha) \hat{D}(\gamma)=\hat{D}(\alpha+\gamma) \mathrm{e}^{\left(\alpha \gamma^{*}-\alpha^{*} \gamma\right) / 2}$. This implies that the displacement operator transforms any coherent state into a coherent one. To be more precise,

$$
\hat{D}\left(\alpha_{1}, \alpha_{2}\right)\left|\gamma_{1}, \gamma_{2}\right\rangle=\hat{D}_{1}\left(\alpha_{1}\right)\left|\gamma_{1}\right\rangle \hat{D}_{2}\left(\alpha_{2}\right)\left|\gamma_{2}\right\rangle=\mathrm{e}^{\left(\alpha_{1} \gamma_{1}^{*}-\alpha_{1}^{*} \gamma_{1}+\alpha_{2} \gamma_{2}^{*}-\alpha_{2}^{*} \gamma_{2}\right) / 2}\left|\alpha_{1}+\gamma_{1}\right\rangle\left|\alpha_{2}+\gamma_{2}\right\rangle .
$$

In much the same way, one can write

$\hat{D}\left(\alpha_{1}, \alpha_{2}\right)\left|-\gamma_{1},-\gamma_{2}\right\rangle=\hat{D}_{1}\left(\alpha_{1}\right)\left|-\gamma_{1}\right\rangle \hat{D}_{2}\left(\alpha_{2}\right)\left|-\gamma_{2}\right\rangle=\mathrm{e}^{\left(\alpha_{1}^{*} \gamma_{1}-\alpha_{1} \gamma_{1}^{*}+\alpha_{2}^{*} \gamma_{2}-\alpha_{2} \gamma_{2}^{*}\right) / 2}\left|\alpha_{1}-\gamma_{1}\right\rangle\left|\alpha_{2}-\gamma_{2}\right\rangle$

The scalar product of the coherent state $|\delta\rangle$ and the Fock basis state $|n\rangle$ is

$$
\langle n \mid \delta\rangle=\langle n|\left(\mathrm{e}^{-|\delta|^{2} / 2} \sum_{k=0}^{\infty} \frac{\delta^{k}}{\sqrt{k !}}|k\rangle\right)=\mathrm{e}^{-|\delta|^{2} / 2} \frac{\delta^{n}}{\sqrt{n !}} .
$$

Using the simplified formula (24) and taking into account the results of Eqs. (21), (23), (25), (26), and (27), we arrive at the following formula for the photon-number tomogram of the state under consideration

$$
\begin{aligned}
& w_{S C}\left(n_{1}, n_{2}, \alpha_{1}, \alpha_{2}\right)=\frac{\mathrm{e}^{-\left(\left|\alpha_{1}\right|^{2}+\left|\alpha_{2}\right|^{2}\right)}}{4 n_{1} ! n_{2} ! \cosh \left(\left|\gamma_{1}\right|^{2}+\left|\gamma_{2}\right|^{2}\right)} \\
& \quad \times\left|\mathrm{e}^{-\left(\alpha_{1}^{*} \gamma_{1}+\alpha_{2}^{*} \gamma_{2}\right)}\left(\alpha_{1}+\gamma_{1}\right)^{n_{1}}\left(\alpha_{2}+\gamma_{2}\right)^{n_{2}}+\mathrm{e}^{\alpha_{1}^{*} \gamma_{1}+\alpha_{2}^{*} \gamma_{2}}\left(\alpha_{1}-\gamma_{1}\right)^{n_{1}}\left(\alpha_{2}-\gamma_{2}\right)^{n_{2}}\right|^{2} .
\end{aligned}
$$

Now, when the photon-number tomogram is known, our goal is to make sure that the Bell-CHSH inequality can be violated, because the state involved is entangled. In Sec. 2, we emphasized that there exist many ways to construct the portrait corresponding to the two-qubit system. In our opinion, the simplest way is to split the tomogram components corresponding to the states:

(i) the vacuum state,

(ii) states with no photons in the first mode and with nonzero number of photons in the second one,

(iii) states with nonzero number of photons in the first mode and with no photons in the second one,

(iv) states with nonzero number of photons in both modes.

Then the probability distribution vector (8) takes the form

$$
\vec{W}_{4 S C}\left(\alpha_{1}, \alpha_{2}\right)=\left(\begin{array}{c}
w\left(0,0, \alpha_{1}, \alpha_{2}\right) \\
\sum_{n_{2}=1}^{\infty} w\left(0, n_{2}, \alpha_{1}, \alpha_{2}\right) \\
\sum_{n_{1}=1}^{\infty} w\left(n_{1}, 0, \alpha_{1}, \alpha_{2}\right) \\
\sum_{n_{1}, n_{2}=1}^{\infty} w\left(n_{1}, n_{2}, \alpha_{1}, \alpha_{2}\right)
\end{array}\right)=\left(\begin{array}{l}
w_{S C}\left(+,+, \alpha_{1}, \alpha_{2}\right) \\
w_{S C}\left(+,-, \alpha_{1}, \alpha_{2}\right) \\
w_{S C}\left(-,+, \alpha_{1}, \alpha_{2}\right) \\
w_{S C}\left(-,-, \alpha_{1}, \alpha_{2}\right)
\end{array}\right)
$$




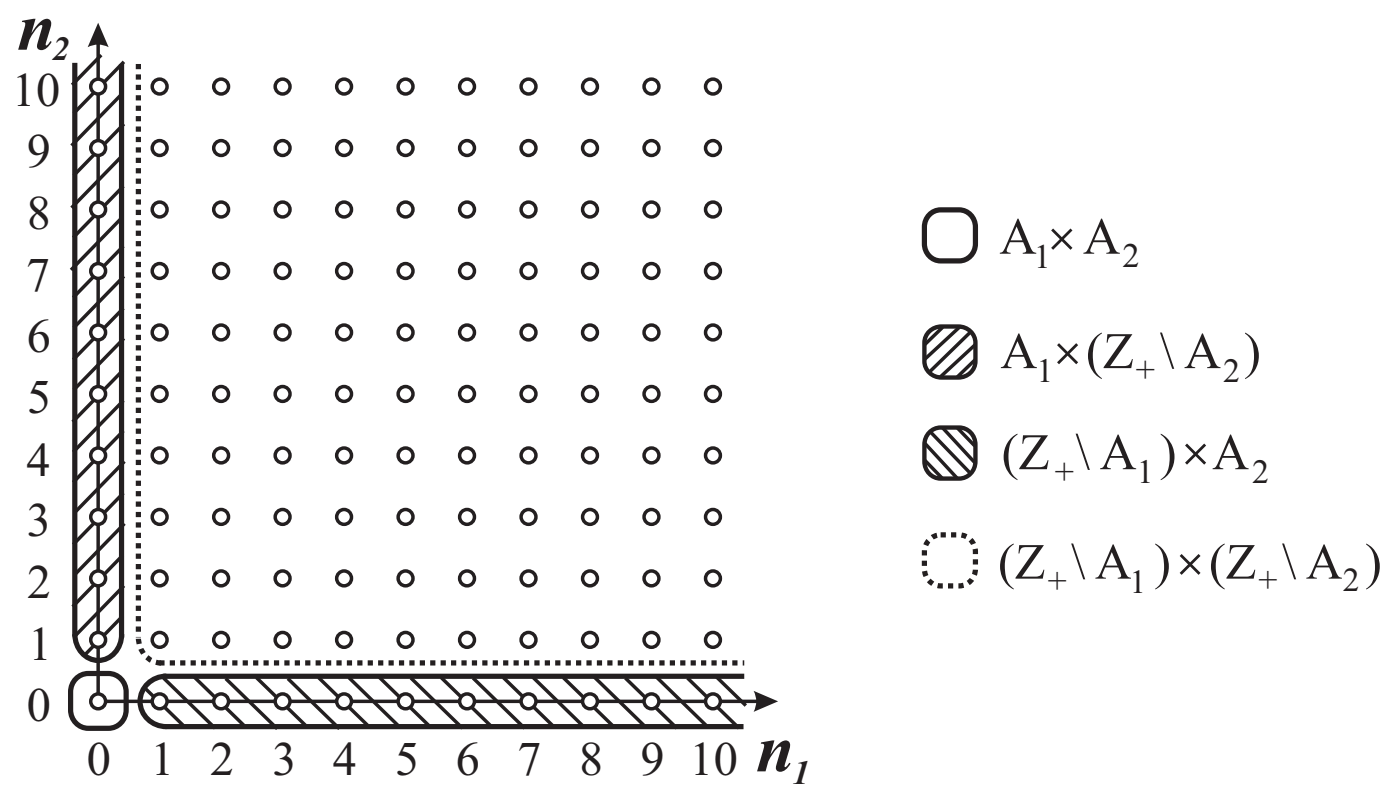

Figure 2: Simplest example of dividing the set $Z_{+} \times Z_{+}$into parts, that enables one to construct the qubit portrait of the photon-number tomogram.

The pattern of such a partition is shown in Fig. 2. Using the explicit expression (28) of the photon-number tomogram for our particular case, we get the components of of the four-vector (29)

$$
\begin{gathered}
w_{S C}\left(+,+, \alpha_{1}, \alpha_{2}\right)=\frac{e^{-\left(\left|\alpha_{1}\right|^{2}+\left|\alpha_{2}\right|^{2}\right)}}{2 \cosh \left(\left|\gamma_{1}\right|^{2}+\left|\gamma_{2}\right|^{2}\right)}\left\{\cosh \left(2 \operatorname{Re}\left(\alpha_{1} \gamma_{1}+\alpha_{2} \gamma_{2}\right)\right)+\cos \left(2 \operatorname{Im}\left(\alpha_{1} \gamma_{1}+\alpha_{2} \gamma_{2}\right)\right)\right\}, \\
w_{S C}\left(+,-, \alpha_{1}, \alpha_{2}\right)=\frac{e^{-\left(\left|\alpha_{1}\right|^{2}+\left|\alpha_{2}\right|^{2}\right)}}{2 \cosh \left(\left|\gamma_{1}\right|^{2}+\left|\gamma_{2}\right|^{2}\right)}\left\{e^{\left|\alpha_{2}\right|^{2}+\left|\gamma_{2}\right|^{2}} \cosh \left(2 \operatorname{Re}\left(\alpha_{1} \gamma_{1}\right)\right)\right. \\
\left.+e^{\left|\alpha_{2}\right|^{2}-\left|\gamma_{2}\right|^{2}} \cos \left(2 \operatorname{Im}\left(\alpha_{1} \gamma_{1}\right)\right)-\cosh \left(2 \operatorname{Re}\left(\alpha_{1} \gamma_{1}+\alpha_{2} \gamma_{2}\right)\right)-\cos \left(2 \operatorname{Im}\left(\alpha_{1} \gamma_{1}+\alpha_{2} \gamma_{2}\right)\right)\right\},(31),(32),(33) \\
w_{S C}\left(-,+, \alpha_{1}, \alpha_{2}\right)=\frac{e^{-\left(\left|\alpha_{1}\right|^{2}+\left|\alpha_{2}\right|^{2}\right)}}{2 \cosh \left(\left|\gamma_{1}\right|^{2}+\left|\gamma_{2}\right|^{2}\right)}\left\{e^{\left|\alpha_{1}\right|^{2}+\left|\gamma_{1}\right|^{2}} \cosh \left(2 \operatorname{Re}\left(\alpha_{2} \gamma_{2}\right)\right)\right. \\
\left.+e^{\left|\alpha_{1}\right|^{2}-\left|\gamma_{1}\right|^{2}} \cos \left(2 \operatorname{Im}\left(\alpha_{2} \gamma_{2}\right)\right)-\cosh \left(2 \operatorname{Re}\left(\alpha_{1} \gamma_{1}+\alpha_{2} \gamma_{2}\right)\right)-\cos \left(2 \operatorname{Im}\left(\alpha_{1} \gamma_{1}+\alpha_{2} \gamma_{2}\right)\right)\right\},(33) \\
w_{S C}\left(-,-, \alpha_{1}, \alpha_{2}\right)=1-w_{S C}\left(+,+, \alpha_{1}, \alpha_{2}\right)-w_{S C}\left(+,-, \alpha_{1}, \alpha_{2}\right)-w_{S C}\left(-,+, \alpha_{1}, \alpha_{2}\right) .
\end{gathered}
$$

Let us now consider the function of four complex variables (or eight real variables) 


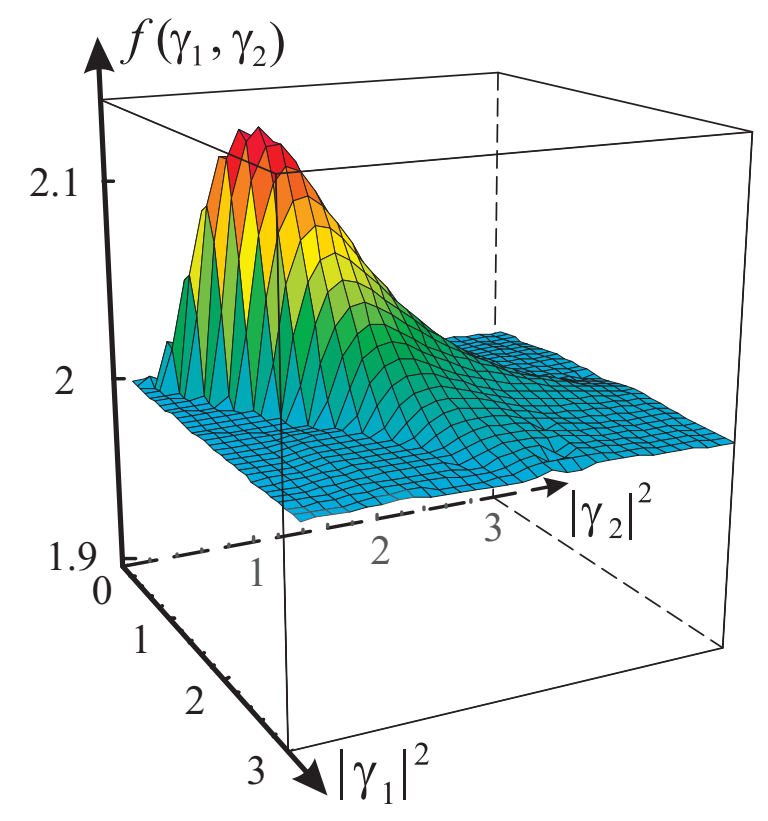

Figure 3: Violation of the Bell-CHSH inequality for the Schrödinger cat states of the form $\left|\gamma_{1}, \gamma_{2}\right\rangle+\left|-\gamma_{1},-\gamma_{2}\right\rangle$. Here the maximum value of the Bell number is denoted as $f\left(\gamma_{1}, \gamma_{2}\right)$. The qubit portrait is constructed using formula (29) ("zero-nonzero" approach).

$$
B_{S C}\left(\alpha_{1}, \alpha_{2}, \beta_{1}, \beta_{2}\right)=\left|\operatorname{Tr}\left(M_{S C}\left(\alpha_{1}, \alpha_{2}, \beta_{1}, \beta_{2}\right) I\right)\right|,
$$

where the matrix $M_{S C}\left(\alpha_{1}, \alpha_{2}, \beta_{1}, \beta_{2}\right)$ is given by the formula

$$
M_{S C}\left(\alpha_{1}, \alpha_{2}, \beta_{1}, \beta_{2}\right)=\left(\vec{W}_{4 S C}\left(\alpha_{1}, \alpha_{2}\right) \quad \vec{W}_{4 S C}\left(\alpha_{1}, \beta_{2}\right) \quad \vec{W}_{4 S C}\left(\beta_{1}, \alpha_{2}\right) \quad \vec{W}_{4 S C}\left(\beta_{1}, \beta_{2}\right)\right)
$$

and the matrix $I$ is determined by Eq. (19).

To demonstrate that there exist such values of variables $\alpha_{1}, \alpha_{2}, \beta_{1}$, and $\beta_{2}$ such that the value of function $B_{S C}\left(\alpha_{1}, \alpha_{2}, \beta_{1}, \beta_{2}\right)$ is greater than 2 , we find the maximum of this function, which depends entirely on the form of the quantum state

$$
f\left(\gamma_{1}, \gamma_{2}\right)=\max _{\alpha_{1}, \alpha_{2}, \beta_{1}, \beta_{2} \in \mathrm{C}} B_{S C}\left(\alpha_{1}, \alpha_{2}, \beta_{1}, \beta_{2}\right)
$$

Numerical calculations show that, whatever values of complex variables $\gamma_{1}$ and $\gamma_{2}$ are taken, the value of function $f\left(\gamma_{1}, \gamma_{2}\right)$ is never less than 2. This fact is depicted in Fig. 3, Nevertheless, the extent to which $f\left(\gamma_{1}, \gamma_{2}\right)$ is greater than 2 differs from one state to the other. If $\left|\gamma_{1}\right| \gg 1$ and $\left|\gamma_{2}\right| \gg 1$, then the efficiency of the technique proposed essentially decreases.

\section{Detecting entanglement of the Gaussian states}

The next example to be investigated is a nonclassical state of light described by the Gaussian density matrix (see, e.g., [28, 33, 37, 48]). It is of some interest because it can characterize 
a two-mode mixed squeezed state. A Gaussian density operator $\hat{\rho}$ of the two-mode light is described by the Wigner function $W\left(p_{1}, p_{2}, q_{1}, q_{2}\right)$ of the form [41]

$$
W\left(p_{1}, p_{2}, q_{1}, q_{2}\right)=\frac{1}{\sqrt{\operatorname{det} \mathbf{M}}} \exp \left\{-\frac{1}{2}(\mathbf{Q}-\langle\mathbf{Q}\rangle) \mathbf{M}^{-\mathbf{1}}(\mathbf{Q}-\langle\mathbf{Q}\rangle)\right\}
$$

where

$$
\mathbf{Q}=\left(\begin{array}{c}
p_{1} \\
p_{2} \\
q_{1} \\
q_{2}
\end{array}\right), \quad \mathbf{M}=\left(\begin{array}{cccc}
\sigma_{p_{1} p_{1}} & \sigma_{p_{1} p_{2}} & \sigma_{p_{1} q_{1}} & \sigma_{p_{1} q_{2}} \\
\sigma_{p_{2} p_{1}} & \sigma_{p_{2} p_{2}} & \sigma_{p_{2} q_{1}} & \sigma_{p_{2} q_{2}} \\
\sigma_{q_{1} p_{1}} & \sigma_{q_{1} p_{2}} & \sigma_{q_{1} q_{1}} & \sigma_{q_{1} q_{2}} \\
\sigma_{q_{2} p_{1}} & \sigma_{q_{2} p_{2}} & \sigma_{q_{2} q_{1}} & \sigma_{q_{2} q_{2}}
\end{array}\right)
$$

Here averaging $\langle A\rangle$ implies $\langle A\rangle=\operatorname{Tr} \hat{\rho} \hat{A}$, where $\hat{A}$ can be one of the operators $\hat{p}_{j}=-i\left(\hat{a}_{j}-\right.$ $\left.\hat{a}_{j}^{\dagger}\right) / \sqrt{2}, \hat{q}_{j}=\left(\hat{a}_{j}+\hat{a}_{j}^{\dagger}\right) / \sqrt{2}, j=1,2$ (variables are assumed to be dimensionless, with $\hbar=1$ ). The components of the real symmetric dispersion matrix $\mathbf{M}$ read

$$
\sigma_{p_{i} p_{j}}=\left\langle\hat{p}_{i} \hat{p}_{j}\right\rangle-\left\langle\hat{p}_{i}\right\rangle\left\langle\hat{p}_{j}\right\rangle, \quad \sigma_{q_{i} q_{j}}=\left\langle\hat{q}_{i} \hat{q}_{j}\right\rangle-\left\langle\hat{q}_{i}\right\rangle\left\langle\hat{q}_{j}\right\rangle, \quad \sigma_{p_{i} q_{j}}=\sigma_{q_{j} p_{i}}=\frac{1}{2}\left\langle\hat{p}_{i} \hat{q}_{j}+\hat{q}_{j} \hat{p}_{i}\right\rangle-\left\langle\hat{p}_{i}\right\rangle\left\langle\hat{q}_{j}\right\rangle
$$

Thus, all the information on the state is contained in the Wigner function (37) depending on 14 real variables (four of them determine the vector $\langle\mathbf{Q}\rangle$ and ten of them define the matrix M).

To obtain the photon-number tomogram (3), it is worth noting that this is merely a photon distribution function of the state $\hat{D}\left(\alpha_{1}, \alpha_{2}\right) \hat{\rho} \hat{D}^{\dagger}\left(\alpha_{1}, \alpha_{2}\right)$. Let us now reveal what is the Wigner function of such a state. For this purpose, we calculate the new average values (see, e.g., [24]). For instance,

$$
\begin{aligned}
\left\langle p_{1}\right\rangle_{\alpha_{1} \alpha_{2}} & =\operatorname{Tr}\left(\hat{D}\left(\alpha_{1}, \alpha_{2}\right) \hat{\rho} \hat{D}^{\dagger}\left(\alpha_{1}, \alpha_{2}\right) \hat{p}_{1}\right) \\
& =\operatorname{Tr}\left(\hat{\rho} \hat{D}_{1}^{\dagger}\left(\alpha_{1}\right) \hat{D}_{2}^{\dagger}\left(\alpha_{2}\right)\left\{-i\left(\hat{a}_{1}-\hat{a}_{1}^{\dagger}\right) / \sqrt{2}\right\} \hat{D}_{1}\left(\alpha_{1}\right) \hat{D}_{2}\left(\alpha_{2}\right)\right) \\
& =\operatorname{Tr}\left(\hat{\rho}\left\{-i\left(\hat{a}_{1}+\alpha_{1}-\hat{a}_{1}^{\dagger}-\alpha_{1}^{*}\right) / \sqrt{2}\right\}\right) \\
& =\operatorname{Tr}\left(\hat{\rho}\left\{\hat{p}_{1}+\sqrt{2} \operatorname{Im} \alpha_{1}\right\}\right)=\left\langle p_{1}\right\rangle+\sqrt{2} \operatorname{Im} \alpha_{1} .
\end{aligned}
$$

Here we used the relation $\hat{D}^{\dagger}(\alpha) \hat{a} \hat{D}(\alpha)=\hat{a}+\alpha \hat{1}$. In a similar way we find $\left\langle p_{2}\right\rangle_{\alpha_{1} \alpha_{2}}=\left\langle p_{2}\right\rangle+$ $\sqrt{2} \operatorname{Im} \alpha_{2},\left\langle q_{1}\right\rangle_{\alpha_{1} \alpha_{2}}=\left\langle q_{1}\right\rangle+\sqrt{2} \operatorname{Re} \alpha_{1}$, and $\left\langle q_{2}\right\rangle_{\alpha_{1} \alpha_{2}}=\left\langle q_{2}\right\rangle+\sqrt{2} \operatorname{Re} \alpha_{2}$. The remarkable fact is that the elements of the matrix $\mathbf{M}$ do not change through a transition from $\hat{\rho}$ to $\hat{D}\left(\alpha_{1}, \alpha_{2}\right) \hat{\rho} \hat{D}^{\dagger}\left(\alpha_{1}, \alpha_{2}\right)$. It follows directly from the formulas (39). We will refer to the vector of new average values as $\langle\mathbf{Q}\rangle_{\alpha_{1} \alpha_{2}}$.

The photon distribution function of a generic $N$-mode mixed Gaussian state of light has been obtained earlier [24, 41, 42]. We use it to compute the photon-number tomogram (3). The result is

$$
w_{G}\left(n_{1}, n_{2}, \alpha_{1}, \alpha_{2}\right)=\frac{\exp \left\{-\langle\mathbf{Q}\rangle_{\alpha_{1} \alpha_{2}}\left(2 \mathbf{M}+\mathbf{I}_{4}\right)^{-1}\langle\mathbf{Q}\rangle_{\alpha_{1} \alpha_{2}}\right\}}{\sqrt{\operatorname{det}\left(\mathbf{M}+\frac{1}{2} \mathbf{I}_{4}\right)}} \frac{H_{n_{1}, n_{2}, n_{1}, n_{2}}^{\{\mathbf{R}\}}}{n_{1} ! n_{2} !},
$$


where $\mathbf{I}_{4}$ is the $4 \times 4$ identity matrix, and the four-dimensional matrix $\mathbf{R}$ and the four-vector $\mathbf{y}$ can be expressed with the help of the matrix

$$
\mathbf{U}=\frac{1}{\sqrt{2}}\left(\begin{array}{cccc}
-i & 0 & i & 0 \\
0 & -i & 0 & i \\
1 & 0 & 1 & 0 \\
0 & 1 & 0 & 1
\end{array}\right)
$$

as

$$
\begin{array}{r}
\mathbf{R}=\mathbf{U}^{\dagger}\left(\mathbf{I}_{4}-2 \mathbf{M}\right)\left(\mathbf{I}_{4}+2 \mathbf{M}\right)^{-1} \mathbf{U}^{*} \\
\mathbf{y}=2 \mathbf{U}^{t r}\left(\mathbf{I}_{4}-2 \mathbf{M}\right)^{-1}\langle\mathbf{Q}\rangle_{\alpha_{1} \alpha_{2}}
\end{array}
$$

and the term of the form $H_{k_{1}, k_{2}, k_{3}, k_{4}}^{\{\mathbf{R}\}}(\mathbf{x})$ is the four-dimensional Hermite polynomial given by the formula [49]

$$
H_{k_{1}, k_{2}, k_{3}, k_{4}}^{\{\mathbf{R}\}}(\mathbf{x})=(-1)^{k_{1}+k_{2}+k_{3}+k_{4}} \exp \left(\frac{1}{2} \mathbf{x R \mathbf { x }}\right) \frac{\partial^{k_{1}+k_{2}+k_{3}+k_{4}}}{\partial x_{1}^{k_{1}} \partial x_{2}^{k_{2}} \partial x_{3}^{k_{3}} \partial x_{4}^{k_{4}}} \exp \left(-\frac{1}{2} \sum_{i, j=1}^{4} R_{i j} x_{i} x_{j}\right)
$$

Once one has the expression of the photon-number tomogram (41), it seems possible to explore the entanglement of the Gaussian states just in the same way as in Sec. 3. By analogy with the previous example, we choose the state which is entangled for sure. For instance, the squeezed state given by its Wigner function of the form

$$
W\left(p_{1}, p_{2}, q_{1}, q_{2}\right)=4 \exp \left\{-2\left(3 p_{1}^{2}-\sqrt{35} p_{1} p_{2}+3 p_{2}^{2}+q_{1}^{2}-\sqrt{3} q_{1} q_{2}+q_{2}^{2}\right)\right\}
$$

is entangled, because it contains the products $p_{1} p_{2}$ and $q_{1} q_{2}$ in the exponent. This state corresponds to the matrix $\mathbf{M}$ and the vector $\langle\mathbf{Q}\rangle$ of the form

$$
\mathbf{M}=\left(\begin{array}{cccc}
3 & \sqrt{35} / 2 & 0 & 0 \\
\sqrt{35} / 2 & 3 & 0 & 0 \\
0 & 0 & 1 & \sqrt{3} / 2 \\
0 & 0 & \sqrt{3} / 2 & 1
\end{array}\right), \quad\langle\mathbf{Q}\rangle=\left(\begin{array}{c}
0 \\
0 \\
0 \\
0
\end{array}\right)
$$

which meets the generalized uncertainty relation $\operatorname{det} \mathbf{M} \geq 1 / 4^{2}$. The substitution of $\mathbf{M}$ for such a matrix in Eqs. (43) and (44) yields

$$
\mathbf{R}=\frac{1}{42}\left(\begin{array}{cccc}
0 & 3 \sqrt{35}-7 \sqrt{3} & 0 & -3 \sqrt{35}-7 \sqrt{3} \\
3 \sqrt{35}-7 \sqrt{3} & 0 & -3 \sqrt{35}-7 \sqrt{3} & 0 \\
0 & -3 \sqrt{35}-7 \sqrt{3} & 0 & 3 \sqrt{35}-7 \sqrt{3} \\
-3 \sqrt{35}-7 \sqrt{3} & 0 & 3 \sqrt{35}-7 \sqrt{3} & 0
\end{array}\right)
$$

and 


$$
\mathbf{y}=\left(\begin{array}{c}
-i\left(\alpha_{1}-\sqrt{\frac{7}{5}} \operatorname{Re} \alpha_{2}\right)-\sqrt{3} \operatorname{Im} \alpha_{2} \\
-i\left(\alpha_{2}-\sqrt{\frac{7}{5}} \operatorname{Re} \alpha_{1}\right)-\sqrt{3} \operatorname{Im} \alpha_{1} \\
i\left(\alpha_{1}^{*}-\sqrt{\frac{7}{5}} \operatorname{Re} \alpha_{2}\right)-\sqrt{3} \operatorname{Im} \alpha_{2} \\
i\left(\alpha_{2}^{*}-\sqrt{\frac{7}{5}} \operatorname{Re} \alpha_{1}\right)-\sqrt{3} \operatorname{Im} \alpha_{1}
\end{array}\right)
$$

Now the photon-number tomogram (41) can be calculated for any given parameters $n_{1}, n_{2}$, $\alpha_{1}$, and $\alpha_{2}$. It is just the right time for trying to detect the entanglement of the state (46)). To start, we apply the approach developed above, which means constructing the probabilitydistribution vector (29), and then the stochastic matrix (35), the trace of the product of which with the matrix (19) leads to the Bell-like number. The obstacle arises during the first step, because the probability-distribution four-vector (29) is hardly computable analytically. The numerical calculation has some particular features concerned with four-dimensional Hermite polynomials. The matter is that it is rather difficult to calculate $H_{n_{1}, n_{2}, n_{1}, n_{2}}^{\{\mathbf{R}\}}(\mathbf{y})$ when $n_{1} \gg 1$ and $n_{2} \gg 1$. Therefore, we deal with numbers $n_{1}$ and $n_{2}$ confined by the ranges $0 \leq n_{1} \leq 30$, $0 \leq n_{2} \leq 30$. To neglect the values of $w_{G}\left(n_{1}, n_{2}, \alpha_{1}, \alpha_{2}\right)$ providing $n_{1}>30$ or $n_{2}>30$ we impose the limitations $\left|\operatorname{Re} \alpha_{i}\right| \leq 2,\left|\operatorname{Im} \alpha_{i}\right| \leq 2, i=1,2$ (and consequently $\left|\operatorname{Re} \beta_{i}\right| \leq 2,\left|\operatorname{Im} \beta_{i}\right| \leq 2$, $i=1,2)$. If this is the case, the total contribution of probabilities $w\left(n_{1}, n_{2}, \alpha_{1}, \alpha_{2}\right)$ with high photon numbers $\left(n_{1}>30, n_{2}>30\right)$ to the distribution vector (29) does not exceed $10^{-4}$ and serves as an error measure. The calculation shows that the Bell-like number (34) is less than 2 providing the variables $\alpha_{1}, \alpha_{2}, \beta_{1}$, and $\beta_{2}$ are not beyond the region specified above.

The problem occurs not due to restrictions introduced but because of the unlucky choice of the qubit-portrait construction. Indeed, during the maximization process the variables $\alpha_{1}$, $\alpha_{2}, \beta_{1}$, and $\beta_{2}$ attempt to distribute in such a way that the quantity $w_{G}\left(n_{1}, n_{2}, \alpha_{1}, \alpha_{2}\right)$ is far from zero when $n_{1} \approx n_{2} \approx 0$, the quantity $w_{G}\left(n_{1}, n_{2}, \alpha_{1}, \beta_{2}\right)$ is far from zero when $n_{1} \approx 0$ and $n_{2} \gg 1$, the quantity $w_{G}\left(n_{1}, n_{2}, \beta_{1}, \alpha_{2}\right)$ is far from zero when $n_{1} \gg 1$ and $n_{2} \approx 0$, and the quantity $w_{G}\left(n_{1}, n_{2}, \beta_{1}, \beta_{2}\right)$ is far from zero when $n_{1} \gg 1$ and $n_{2} \gg 1$. In Fig. 2, one can see that the narrowness of the domains $B_{2}$ and $B_{3}$ prevents the quantities $\sum_{n_{2}=1}^{\infty} w\left(0, n_{2}, \alpha_{1}, \beta_{2}\right)$ and $\sum_{n_{1}=1}^{\infty} w\left(n_{1}, 0, \beta_{1}, \alpha_{2}\right)$ from increasing. In our case they cannot be greater then $2 / 3$. (See analogues problem discussed in [50])

To put the situation right we suggest the other separation $\left\{A_{1} \times A_{2}, A_{1} \times\left(Z_{+} \backslash A_{2}\right),\left(Z_{+} \backslash A_{1}\right) \times\right.$ $\left.A_{2},\left(Z_{+} \backslash A_{1}\right) \times\left(Z_{+} \backslash A_{2}\right)\right\}$ of the set $Z_{+} \times Z_{+}$, which is needed to construct the four-dimensional probability-distribution vector. The main idea of the partitioning proposed is patterned in Fig. 4. It implies distinguishing even and odd numbers of photons in individual modes. Such a choice is stimulated by the peculiarities of the photon-distribution function of squeezed states concerned with its oscillations [52, 51]. Thus, the explicit expression of the four-vector $\vec{M}_{4} G$ reads 


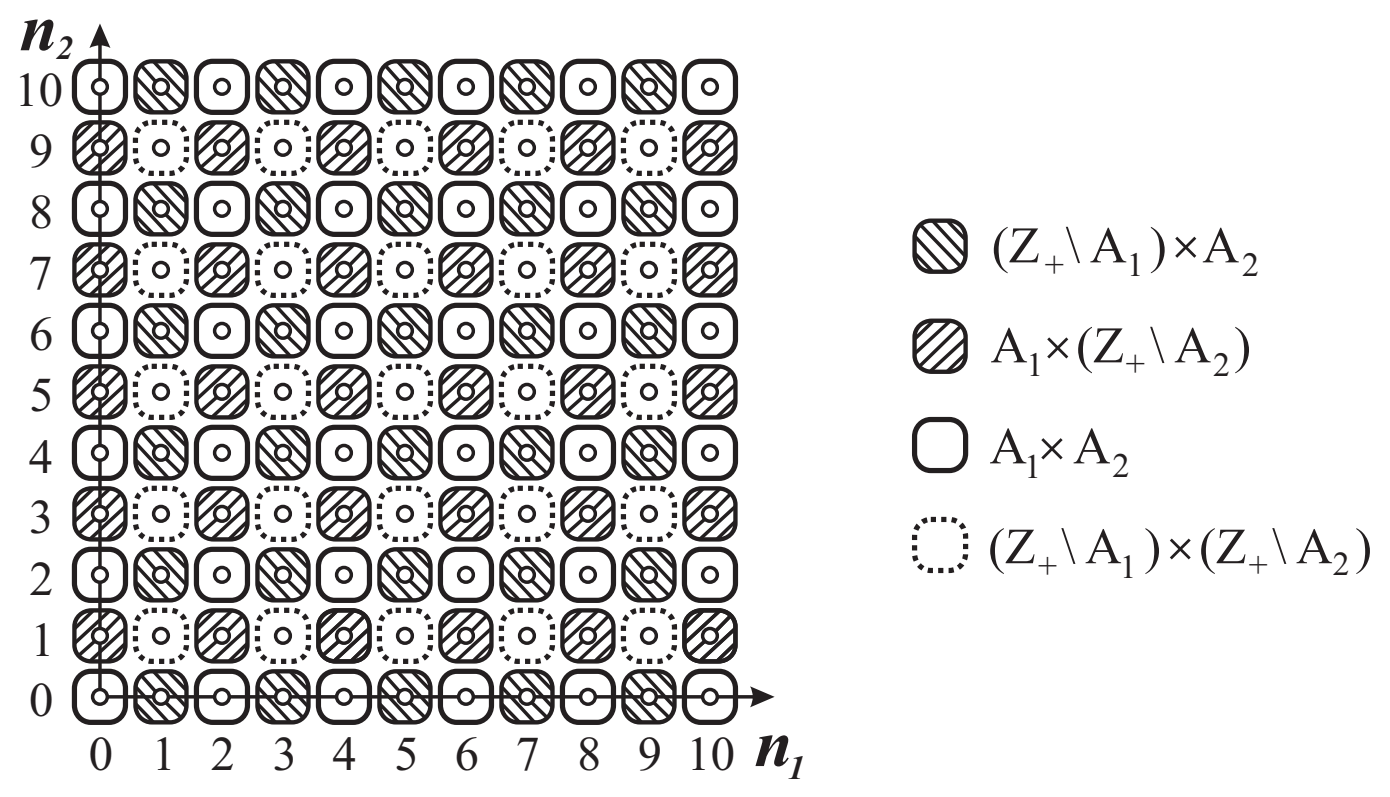

Figure 4: Alternative partition of the set $Z_{+} \times Z_{+}$. The sets $A_{1}$ and $A_{2}$ are nonnegative even integers, while the sets $Z_{+} \backslash A_{1}$ and $Z_{+} \backslash A_{2}$ are nonnegative odd integers.

$$
\vec{W}_{4 G}\left(\alpha_{1}, \alpha_{2}\right)=\left(\begin{array}{c}
\sum_{n_{1}=0}^{\infty} \sum_{n_{2}=0}^{\infty} w\left(2 n_{1}, 2 n_{2}, \alpha_{1}, \alpha_{2}\right) \\
\sum_{n_{1}=0}^{\infty} \sum_{n_{2}=0}^{\infty} w\left(2 n_{1}, 2 n_{2}+1, \alpha_{1}, \alpha_{2}\right) \\
\sum_{n_{1}=0}^{\infty} \sum_{n_{2}=0}^{\infty} w\left(2 n_{1}+1,2 n_{2}, \alpha_{1}, \alpha_{2}\right) \\
\sum_{n_{1}=0}^{\infty} \sum_{n_{2}=0}^{\infty} w\left(2 n_{1}+1,2 n_{2}+1, \alpha_{1}, \alpha_{2}\right)
\end{array}\right)=\left(\begin{array}{c}
w_{G}\left(+,+, \alpha_{1}, \alpha_{2}\right) \\
w_{G}\left(+,-, \alpha_{1}, \alpha_{2}\right) \\
w_{G}\left(-,+, \alpha_{1}, \alpha_{2}\right) \\
w_{G}\left(-,-, \alpha_{1}, \alpha_{2}\right)
\end{array}\right)
$$

The matrix $M_{G}\left(\alpha_{1}, \alpha_{2}, \beta_{1}, \beta_{2}\right)$ appears naturally in the same way as the matrix (35). To demonstrate that the inequality $\left|\operatorname{Tr}\left(M_{G}\left(\alpha_{1}, \alpha_{2}, \beta_{1}, \beta_{2}\right) I\right)\right| \leq 2$ can be violated, it is enough to make the substitution $\alpha_{1}=-0.12 i, \alpha_{2}=0.04 i, \beta_{1}=0.22 i, \beta_{2}=-0.32 i$. Then the matrix $M_{G}$ takes the form

$$
M_{G}(-0.12 i, 0.04 i, 0.22 i,-0.32 i)=\left(\begin{array}{cccc}
0.6199 & 0.5907 & 0.6083 & 0.4678 \\
0.0222 & 0.0515 & 0.0291 & 0.1696 \\
0.0241 & 0.0395 & 0.0357 & 0.1624 \\
0.3335 & 0.3181 & 0.3266 & 0.2000
\end{array}\right)
$$

and $\left|\operatorname{Tr}\left(M_{G}(-0.12 i, 0.04 i, 0.22 i,-0.32 i) I\right)\right| \approx 2.26>2$. The violation of the Bell-CHSH inequality for this particular set of variables $\alpha_{1}, \alpha_{2}, \beta_{1}$, and $\beta_{2}$ indicates immediately that the Gaussian state (46) is entangled.

While dealing with Gaussian states, one should notice that the presented method of tomogram calculation works well for both pure and mixed states. This is accompanied by the 


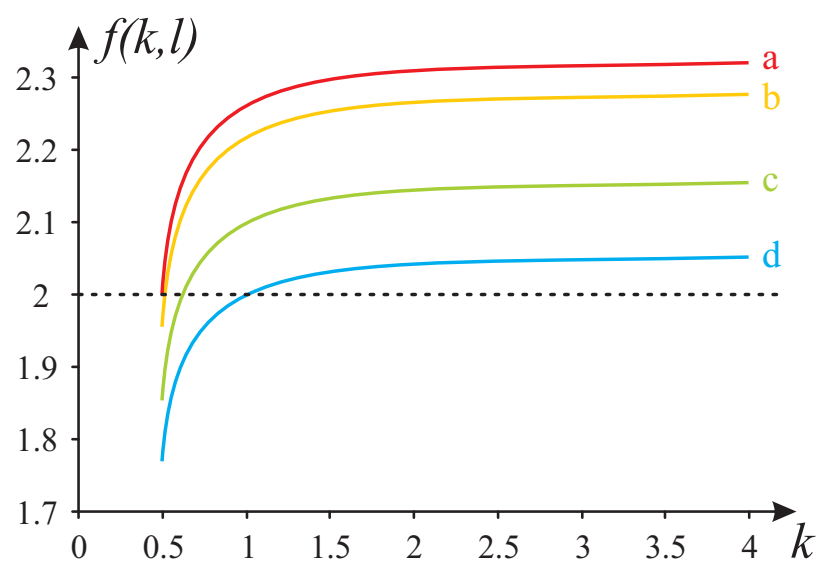

Figure 5: Violation of the Bell-CHSH inequality for the family of states (52). The maximum of the Bell-like number is denoted by $f(k, l)$. The purity of states does not change while moving along the separate curve: pure states with $l=0$ (a), mixed states with $l=0.01$ (b), $l=0.04$ $(\mathrm{c})$, and $l=0.07(\mathrm{~d})$.

complexity of calculations including multidimensional Hermite polynomial contrast to the relatively easy ones for pure states. To demonstrate the advantages of the technique proposed, we consider the family of generally mixed states given by matrix $\mathbf{M}$ and the vector $\mathbf{Q}$ of the form

$$
\mathbf{M}(k, l)=\left(\begin{array}{cccc}
k+\frac{l}{k} & \sqrt{k^{2}-\frac{1}{4}} & 0 & 0 \\
\sqrt{k^{2}-\frac{1}{4}} & k & 0 & 0 \\
0 & 0 & k & \sqrt{k^{2}-\frac{1}{4}} \\
0 & 0 & \sqrt{k^{2}-\frac{1}{4}} & k
\end{array}\right), \quad\langle\mathbf{Q}\rangle=\left(\begin{array}{l}
0 \\
0 \\
0 \\
0
\end{array}\right) .
$$

The purity of this state is determined by the parameter $l$ only, because $\operatorname{det} \mathbf{M}=(1+4 l) / 16$. Thus the state is pure when $l=0$ and mixed for all $l>0$. Denoting

$$
f(k, l)=\max _{\alpha_{1}, \alpha_{2}, \beta_{1}, \beta_{2} \in \mathrm{C}} \operatorname{Tr}\left(B\left(\alpha_{1}, \alpha_{2}, \beta_{1}, \beta_{2}\right) I\right)
$$

we succeeded in plotting the dependence of $f(k, l)$ on $k$ for the states with different purities (see Fig. 5). Though the violation of the Bell-CHSH inequality is clearly observed for pure states, it is rather difficult to detect entanglement of mixed states. This fact is similar to the two-qubit system behaviour when the increase in mixing causes the loss of entanglement.

We have been just convinced that the technique of constructing the qubit portrait proposed in this section can be efficient enough for detecting entanglement of Gaussian states. To check if it works properly for other kinds of states, we apply it to the Schrödinger cat state discussed in the previous section. Though the photon-number tomogram of such a state can be obtained by means of addition of probability distribution functions of Gaussian states, we are going to exploit the explicit formula (28).

Then the even-odd photon number form of the probability distribution vector (50) reads 


$$
\begin{aligned}
& w\left(+,+, \alpha_{1}, \alpha_{2}\right)=\frac{\mathrm{e}^{-\left(\left|\alpha_{1}\right|^{2}+\left|\alpha_{2}\right|^{2}\right)}}{4 \cosh \left(\left|\gamma_{1}\right|^{2}+\left|\gamma_{2}\right|^{2}\right)} \times\left\{\mathrm{e}^{-2 \operatorname{Re}\left(\alpha_{1}^{*} \gamma_{1}+\alpha_{2}^{*} \gamma_{2}\right)} \cosh \left(\left|\alpha_{1}+\gamma_{1}\right|^{2}\right) \cosh \left(\left|\alpha_{2}+\gamma_{2}\right|^{2}\right)\right. \\
& +\mathrm{e}^{2 \operatorname{Re}\left(\alpha_{1}^{*} \gamma_{1}+\alpha_{2}^{*} \gamma_{2}\right)} \cosh \left(\left|\alpha_{1}-\gamma_{1}\right|^{2}\right) \cosh \left(\left|\alpha_{2}-\gamma_{2}\right|^{2}\right) \\
& +2 \cos \left(2 \operatorname{Im}\left(\alpha_{1}^{*} \gamma_{1}+\alpha_{2}^{*} \gamma_{2}\right)\right) \\
& \times\left[\cosh \left(\left|\alpha_{1}\right|^{2}-\left|\gamma_{1}\right|^{2}\right) \cosh \left(\left|\alpha_{2}\right|^{2}-\left|\gamma_{2}\right|^{2}\right) \cos \left(2 \operatorname{Im}\left(\alpha_{1}^{*} \gamma_{1}\right)\right) \cos \left(2 \operatorname{Im}\left(\alpha_{2}^{*} \gamma_{2}\right)\right)\right. \\
& \left.-\sinh \left(\left|\alpha_{1}\right|^{2}-\left|\gamma_{1}\right|^{2}\right) \sinh \left(\left|\alpha_{2}\right|^{2}-\left|\gamma_{2}\right|^{2}\right) \sin \left(2 \operatorname{Im}\left(\alpha_{1}^{*} \gamma_{1}\right)\right) \sin \left(2 \operatorname{Im}\left(\alpha_{2}^{*} \gamma_{2}\right)\right)\right] \\
& +2 \sin \left(2 \operatorname{Im}\left(\alpha_{1}^{*} \gamma_{1}+\alpha_{2}^{*} \gamma_{2}\right)\right) \\
& \times\left[\cosh \left(\left|\alpha_{1}\right|^{2}-\left|\gamma_{1}\right|^{2}\right) \sinh \left(\left|\alpha_{2}\right|^{2}-\left|\gamma_{2}\right|^{2}\right) \cos \left(2 \operatorname{Im}\left(\alpha_{1}^{*} \gamma_{1}\right)\right) \sin \left(2 \operatorname{Im}\left(\alpha_{2}^{*} \gamma_{2}\right)\right)\right. \\
& \left.\left.+\sinh \left(\left|\alpha_{1}\right|^{2}-\left|\gamma_{1}\right|^{2}\right) \cosh \left(\left|\alpha_{2}\right|^{2}-\left|\gamma_{2}\right|^{2}\right) \sin \left(2 \operatorname{Im}\left(\alpha_{1}^{*} \gamma_{1}\right)\right) \cos \left(2 \operatorname{Im}\left(\alpha_{2}^{*} \gamma_{2}\right)\right)\right]\right\}, \\
& w\left(+,-, \alpha_{1}, \alpha_{2}\right)=\frac{\mathrm{e}^{-\left(\left|\alpha_{1}\right|^{2}+\left|\alpha_{2}\right|^{2}\right)}}{4 \cosh \left(\left|\gamma_{1}\right|^{2}+\left|\gamma_{2}\right|^{2}\right)} \times\left\{\mathrm{e}^{-2 \operatorname{Re}\left(\alpha_{1}^{*} \gamma_{1}+\alpha_{2}^{*} \gamma_{2}\right)} \cosh \left(\left|\alpha_{1}+\gamma_{1}\right|^{2}\right) \sinh \left(\left|\alpha_{2}+\gamma_{2}\right|^{2}\right)\right. \\
& +\mathrm{e}^{2 \operatorname{Re}\left(\alpha_{1}^{*} \gamma_{1}+\alpha_{2}^{*} \gamma_{2}\right)} \cosh \left(\left|\alpha_{1}-\gamma_{1}\right|^{2}\right) \sinh \left(\left|\alpha_{2}-\gamma_{2}\right|^{2}\right) \\
& +2 \cos \left(2 \operatorname{Im}\left(\alpha_{1}^{*} \gamma_{1}+\alpha_{2}^{*} \gamma_{2}\right)\right) \\
& \times\left[\cosh \left(\left|\alpha_{1}\right|^{2}-\left|\gamma_{1}\right|^{2}\right) \sinh \left(\left|\alpha_{2}\right|^{2}-\left|\gamma_{2}\right|^{2}\right) \cos \left(2 \operatorname{Im}\left(\alpha_{1}^{*} \gamma_{1}\right)\right) \cos \left(2 \operatorname{Im}\left(\alpha_{2}^{*} \gamma_{2}\right)\right)\right. \\
& \left.-\sinh \left(\left|\alpha_{1}\right|^{2}-\left|\gamma_{1}\right|^{2}\right) \cosh \left(\left|\alpha_{2}\right|^{2}-\left|\gamma_{2}\right|^{2}\right) \sin \left(2 \operatorname{Im}\left(\alpha_{1}^{*} \gamma_{1}\right)\right) \sin \left(2 \operatorname{Im}\left(\alpha_{2}^{*} \gamma_{2}\right)\right)\right] \\
& +2 \sin \left(2 \operatorname{Im}\left(\alpha_{1}^{*} \gamma_{1}+\alpha_{2}^{*} \gamma_{2}\right)\right) \\
& \times\left[\cosh \left(\left|\alpha_{1}\right|^{2}-\left|\gamma_{1}\right|^{2}\right) \cosh \left(\left|\alpha_{2}\right|^{2}-\left|\gamma_{2}\right|^{2}\right) \cos \left(2 \operatorname{Im}\left(\alpha_{1}^{*} \gamma_{1}\right)\right) \sin \left(2 \operatorname{Im}\left(\alpha_{2}^{*} \gamma_{2}\right)\right)\right. \\
& \left.\left.+\sinh \left(\left|\alpha_{1}\right|^{2}-\left|\gamma_{1}\right|^{2}\right) \sinh \left(\left|\alpha_{2}\right|^{2}-\left|\gamma_{2}\right|^{2}\right) \sin \left(2 \operatorname{Im}\left(\alpha_{1}^{*} \gamma_{1}\right)\right) \cos \left(2 \operatorname{Im}\left(\alpha_{2}^{*} \gamma_{2}\right)\right)\right]\right\} \\
& w\left(-,+, \alpha_{1}, \alpha_{2}\right)=\frac{\mathrm{e}^{-\left(\left|\alpha_{1}\right|^{2}+\left|\alpha_{2}\right|^{2}\right)}}{4 \cosh \left(\left|\gamma_{1}\right|^{2}+\left|\gamma_{2}\right|^{2}\right)} \times\left\{\mathrm{e}^{-2 \operatorname{Re}\left(\alpha_{1}^{*} \gamma_{1}+\alpha_{2}^{*} \gamma_{2}\right)} \sinh \left(\left|\alpha_{1}+\gamma_{1}\right|^{2}\right) \cosh \left(\left|\alpha_{2}+\gamma_{2}\right|^{2}\right)\right. \\
& +\mathrm{e}^{2 \operatorname{Re}\left(\alpha_{1}^{*} \gamma_{1}+\alpha_{2}^{*} \gamma_{2}\right)} \sinh \left(\left|\alpha_{1}-\gamma_{1}\right|^{2}\right) \cosh \left(\left|\alpha_{2}-\gamma_{2}\right|^{2}\right) \\
& +2 \cos \left(2 \operatorname{Im}\left(\alpha_{1}^{*} \gamma_{1}+\alpha_{2}^{*} \gamma_{2}\right)\right) \\
& \times\left[\sinh \left(\left|\alpha_{1}\right|^{2}-\left|\gamma_{1}\right|^{2}\right) \cosh \left(\left|\alpha_{2}\right|^{2}-\left|\gamma_{2}\right|^{2}\right) \cos \left(2 \operatorname{Im}\left(\alpha_{1}^{*} \gamma_{1}\right)\right) \cos \left(2 \operatorname{Im}\left(\alpha_{2}^{*} \gamma_{2}\right)\right)\right. \\
& \left.-\cosh \left(\left|\alpha_{1}\right|^{2}-\left|\gamma_{1}\right|^{2}\right) \sinh \left(\left|\alpha_{2}\right|^{2}-\left|\gamma_{2}\right|^{2}\right) \sin \left(2 \operatorname{Im}\left(\alpha_{1}^{*} \gamma_{1}\right)\right) \sin \left(2 \operatorname{Im}\left(\alpha_{2}^{*} \gamma_{2}\right)\right)\right] \\
& +2 \sin \left(2 \operatorname{Im}\left(\alpha_{1}^{*} \gamma_{1}+\alpha_{2}^{*} \gamma_{2}\right)\right) \\
& \times\left[\cosh \left(\left|\alpha_{1}\right|^{2}-\left|\gamma_{1}\right|^{2}\right) \cosh \left(\left|\alpha_{2}\right|^{2}-\left|\gamma_{2}\right|^{2}\right) \sin \left(2 \operatorname{Im}\left(\alpha_{1}^{*} \gamma_{1}\right)\right) \cos \left(2 \operatorname{Im}\left(\alpha_{2}^{*} \gamma_{2}\right)\right)\right.
\end{aligned}
$$




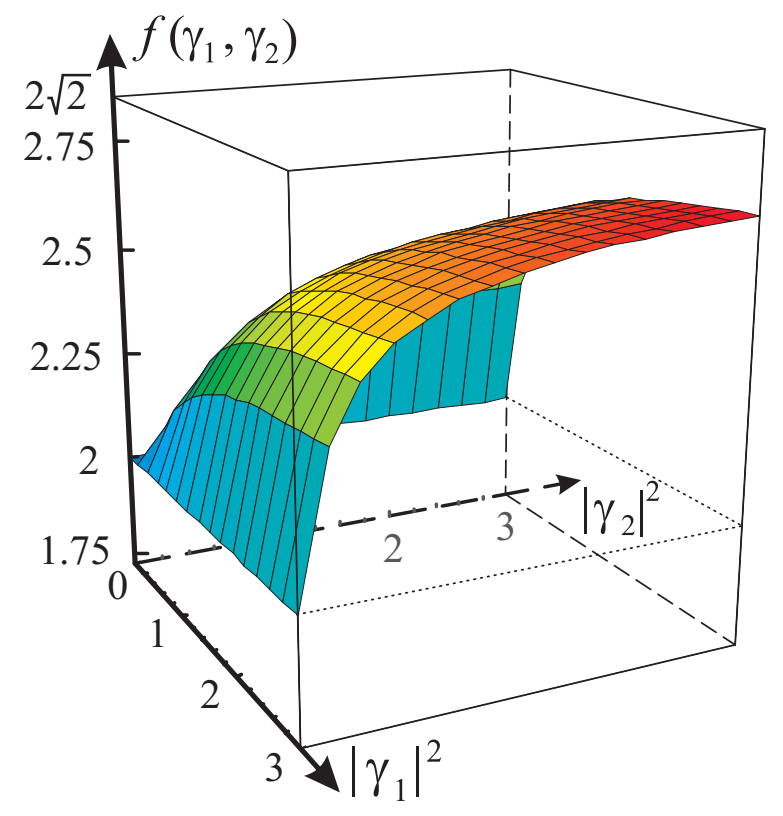

Figure 6: Violation of the Bell-CHSH inequality for the Schrödinger cat states of the form $\left|\gamma_{1}, \gamma_{2}\right\rangle+\left|-\gamma_{1},-\gamma_{2}\right\rangle$. Here the maximum value of the Bell-like number is denoted by $f\left(\gamma_{1}, \gamma_{2}\right)$. The qubit portrait is constructed using formula (50) ("even-odd" approach).

$$
\begin{gathered}
\left.\left.+\sinh \left(\left|\alpha_{1}\right|^{2}-\left|\gamma_{1}\right|^{2}\right) \sinh \left(\left|\alpha_{2}\right|^{2}-\left|\gamma_{2}\right|^{2}\right) \cos \left(2 \operatorname{Im}\left(\alpha_{1}^{*} \gamma_{1}\right)\right) \sin \left(2 \operatorname{Im}\left(\alpha_{2}^{*} \gamma_{2}\right)\right)\right]\right\} \\
w\left(-,-, \alpha_{1}, \alpha_{2}\right)=\frac{\mathrm{e}^{-\left(\left|\alpha_{1}\right|^{2}+\left|\alpha_{2}\right|^{2}\right)}}{4 \cosh \left(\left|\gamma_{1}\right|^{2}+\left|\gamma_{2}\right|^{2}\right)} \times\left\{\mathrm{e}^{-2 \operatorname{Re}\left(\alpha_{1}^{*} \gamma_{1}+\alpha_{2}^{*} \gamma_{2}\right)} \sinh \left(\left|\alpha_{1}+\gamma_{1}\right|^{2}\right) \sinh \left(\left|\alpha_{2}+\gamma_{2}\right|^{2}\right)\right. \\
+\mathrm{e}^{2 \operatorname{Re}\left(\alpha_{1}^{*} \gamma_{1}+\alpha_{2}^{*} \gamma_{2}\right)} \sinh \left(\left|\alpha_{1}-\gamma_{1}\right|^{2}\right) \sinh \left(\left|\alpha_{2}-\gamma_{2}\right|^{2}\right) \\
+2 \cos \left(2 \operatorname{Im}\left(\alpha_{1}^{*} \gamma_{1}+\alpha_{2}^{*} \gamma_{2}\right)\right) \\
\times\left[\sinh \left(\left|\alpha_{1}\right|^{2}-\left|\gamma_{1}\right|^{2}\right) \sinh \left(\left|\alpha_{2}\right|^{2}-\left|\gamma_{2}\right|^{2}\right) \cos \left(2 \operatorname{Im}\left(\alpha_{1}^{*} \gamma_{1}\right)\right) \cos \left(2 \operatorname{Im}\left(\alpha_{2}^{*} \gamma_{2}\right)\right)\right. \\
\left.-\cosh \left(\left|\alpha_{1}\right|^{2}-\left|\gamma_{1}\right|^{2}\right) \cosh \left(\left|\alpha_{2}\right|^{2}-\left|\gamma_{2}\right|^{2}\right) \sin \left(2 \operatorname{Im}\left(\alpha_{1}^{*} \gamma_{1}\right)\right) \sin \left(2 \operatorname{Im}\left(\alpha_{2}^{*} \gamma_{2}\right)\right)\right] \\
+2 \sin \left(2 \operatorname{Im}\left(\alpha_{1}^{*} \gamma_{1}+\alpha_{2}^{*} \gamma_{2}\right)\right) \\
\times\left[\sinh \left(\left|\alpha_{1}\right|^{2}-\left|\gamma_{1}\right|^{2}\right) \cosh \left(\left|\alpha_{2}\right|^{2}-\left|\gamma_{2}\right|^{2}\right) \cos \left(2 \operatorname{Im}\left(\alpha_{1}^{*} \gamma_{1}\right)\right) \sin \left(2 \operatorname{Im}\left(\alpha_{2}^{*} \gamma_{2}\right)\right)\right. \\
\left.\left.+\cosh \left(\left|\alpha_{1}\right|^{2}-\left|\gamma_{1}\right|^{2}\right) \sinh \left(\left|\alpha_{2}\right|^{2}-\left|\gamma_{2}\right|^{2}\right) \sin \left(2 \operatorname{Im}\left(\alpha_{1}^{*} \gamma_{1}\right)\right) \cos \left(2 \operatorname{Im}\left(\alpha_{2}^{*} \gamma_{2}\right)\right)\right]\right\}
\end{gathered}
$$

By a method analogues to that used in Sec. 3, we calculate the maximum of the Bell-like number $B_{S C}\left(\alpha_{1}, \alpha_{2}, \beta_{1}, \beta_{2}\right)=\left|\operatorname{Tr}\left(M_{S C}\left(\alpha_{1}, \alpha_{2}, \beta_{1}, \beta_{2}\right) I\right)\right|$ over the complex numbers $\alpha_{1}, \alpha_{2}, \beta_{1}$, and $\beta_{2}$, which is a function depending entirely on the form of the quantum state

$$
f\left(\gamma_{1}, \gamma_{2}\right)=\max _{\alpha_{1}, \alpha_{2}, \beta_{1}, \beta_{2} \in C} B_{S C}\left(\alpha_{1}, \alpha_{2}, \beta_{1}, \beta_{2}\right) .
$$


The numerical calculation of this function for real numbers $\gamma_{1}$ and $\gamma_{2}$ is shown in Fig. 6. Comparison of Fig. [6 with Fig. 3 apparently shows that the "even-odd" approach of constructing the qubit portrait achieves better results than the "zero-nonzero" method.

In Fig. 6, we can see that the Schrödinger cat state (20) is always entangled except the cases $\gamma_{1}=0$ or $\gamma_{2}=0$, where it becomes separable $|0\rangle\left(\left|\gamma_{2}\right\rangle+\left|-\gamma_{2}\right\rangle\right)$ or $\left(\left|\gamma_{1}\right\rangle+\left|-\gamma_{1}\right\rangle\right)|0\rangle$. While moving to large $\left|\gamma_{1}\right|$ and $\left|\gamma_{1}\right|$, the function $f\left(\gamma_{1}, \gamma_{2}\right)$ increases. This increase can be ascribed to the fact that if $\left|\gamma_{i}\right| \gg 1$, the states $\left|\gamma_{i}\right\rangle$ and $\left|-\gamma_{i}\right\rangle$ become quasiorthogonal due to $\left|\left\langle\gamma_{i} \mid-\gamma_{i}\right\rangle\right| \ll 1$. If this is the case, the state (20) resembles the usual two-qubit cat state $\frac{1}{\sqrt{2}}(|00\rangle+|11\rangle)$, which is maximally entangled and leads to the greatest violation of the Bell inequality $B=2 \sqrt{2}$ (also known as the Cirelson bound [53]). As far as our case of two-mode light state is concerned, the function $f\left(\gamma_{1}, \gamma_{2}\right)$ equals approximately 2.7 when $\gamma_{1}=\gamma_{2}=10$, equals approximately 2.78 when $\gamma_{1}=\gamma_{2}=50$, and seems to tend to $2 \sqrt{2}$ when $\left|\gamma_{1}\right|$ and $\left|\gamma_{2}\right|$ go to infinity.

\section{Conclusions}

The photon-number tomogram is similar to the qudit tomogram (i.e., spin tomogram of the system with the total spin $j$ ), because of the countability of the outputs. The conventional approach of constructing the qubit portrait of qudit states can be applied to the pnoton number tomogram with some modifications. Being as powerful as its qudit counterpart, such a method suits for detecting entanglement of two-mode light states. To be more exact, the violation of the Bell-CHSH inequality indicates immediately that the state is entangled. Since there are many ways of reducing the photon-number tomogram of the two-mode light state to the spin tomogram of the two-qubit system, the effectiveness of the entanglement detection depends on the method utilized. To illustrate this fact we compared two of them in this paper.

\section{Acknowledgments}

V. I. M. thanks the Russian Foundation for Basic Research for partial support under Project Nos. 07-02-00598 and 08-02-90300.

\section{References}

[1] S. Mancini, V. I. Man'ko, and P. Tombesi, Phys. Lett. A, 213, 1 (1996).

[2] J. Bertrand and P. Bertrand, Found. Phys., 17, 397 (1987).

[3] K. Vogel and H. Risken, Phys. Rev. A, 40, 2847 (1989).

[4] E. P. Wigner, Phys. Rev., 40, 749 (1932).

[5] D. T. Smithey, M. Beck, M. G. Raymer, and A. Faridani, Phys. Rev. Lett., 70, 1244 (1993).

[6] V. I. Man'ko and R. V. Mendes, Physica D, 145, 330 (2000). 
[7] O. Man'ko and V. I. Man'ko, J. Russ. Laser Res., 18, 407 (1997).

[8] O. V. Man'ko, V. I. Man'ko, and G. Marmo, J. Phys. A: Math. Gen., 35, 699 (2002).

[9] V. I. Man'ko, G. Marmo, A. Simoni, E. C. G. Sudarshan, and F. Ventrigilia, Rep. Math. Phys., 61, 337 (2008).

[10] V. V. Dodonov and V. I. Man'ko, Phys. Lett. A, 229, 335 (1997).

[11] V. I. Man'ko and O. V. Man'ko, J. Exp. Ther. Phys., 85, 430 (1997).

[12] E. Schrödinger, Ann. d. Phys., Lpz., 79, 489 (1926).

[13] R. P. Feynmann, Rev. Mod. Phys., 20, 367 (1948).

[14] J. E. Moyal, Proc. Cambridge Philos. Soc., 45, 99 (1949).

[15] C. Lupo, V. I. Man'ko, and G. Marmo, J. Phys. A: Math. Theor., 40, 13091 (2007).

[16] C. Lupo, V. I. Man'ko, and G. Marmo, J. Phys. A: Math. Gen., 39, 12515 (2006).

[17] C. Lupo, V. I. Man'ko, G. Marmo, and E. C. G. Sudarshan, J. Phys. A: Math. Gen., 38, 10377 (2005).

[18] V. N. Chernega and V. I. Man'ko, J. Russ. Laser Res., 28, 103 (2007).

[19] S. Mancini, V. I. Man'ko, E. V. Shchukin, and P. Tombesi, J. Opt. B: Quantum Semiclass. Opt., 5, 333 (2003).

[20] A. A. Kolesnikov, M. A. Anisimov and V. I. Man'ko, "Tomographic approach to the Bell's inequality for two-, three- and four-mode light states," Proceedings of the 51st Conference of the Moscow Institute of Physics and Technology (Dolgoprudnyi, Moscow Region, Russia, November 2008), MIPT Publishers, Moscow, Russia (2008), Vol. 8, p. 172 [in Russian].

[21] K. Banaszek and K. Wodkiewicz, Phys. Rev. Lett., 76, 4344 (1996).

[22] S. Wallentowitz and W. Vogel, Phys. Rev. A, 53, 4528 (1996).

[23] S. Mancini, P. Tombesi, and V. I. Man'ko, Europhys. Lett., 37, 79 (1997).

[24] O. Man'ko and V. I. Man'ko, J. Russ. Laser Res., 24, 497 (2003).

[25] E. C. G. Sudarshan, P. M. Mathews, and J. Rau, Phys. Rev., 121, 920 (1961).

[26] M. Horodecki, P. Horodecki, and R. Horodecki, Phys. Lett. A, 223, 1 (1996).

[27] A. Peres, Phys. Rev. Lett., 77, 1413 (1996).

[28] R. Simon, Phys. Rev. Lett., 84, 2726 (2000).

[29] O. V. Man'ko, V. I. Man'ko, G. Marmo, A. Shaji, E. C. G. Sudarshan, and F. Zaccaria, Phys. Lett. A, 339, 194 (2005). 
[30] O. V. Man'ko, V. I. Man'ko, E. C. G. Sudarshan, and F. Zaccaria, Phys. Lett. A, 357, 255 (2006).

[31] A. S. Chirkin and M. Yu. Saigin, Acta Phys. Hung. B, 26/1-2, 63 (2006).

[32] M. Yu. Saigin and A. S. Chirkin, Mod. Probl. Stat. Phys., 5, 169 (2006).

[33] A. S. Chirkin and M. Yu. Saigin, J. Russ. Laser Res., 28, 505 (2007).

[34] V. V. Dodonov, J. Opt. B: Quantum Semiclass. Opt., 4, R1 (2002).

[35] V. V. Dodonov and V. I. Man'ko, Theory of Nonclassical States of Light, Taylor \& Francis, London (2003).

[36] S. N. Filippov and V. I. Man'ko, J. Russ. Laser Res., 29, 564 (2008).

[37] A. Serafini, G. Adesso, and F. Illuminati, Phys. Rev. A, 71, 023249, (2005).

[38] J. S. Bell, Physics, 1, 195 (1964).

[39] J. F. Clauser, M. A. Horne, A. Shimony, and R. A. Holt, Phys. Rev. Lett., 23, 880 (1969).

[40] G. M. D'Ariano, S. Mancini, V. I. Man'ko, and P. Tombesi, Quantum Semicl. Optics, 8, 1017, (1996).

[41] V. V. Dodonov, O. V. Man'ko, and V. I. Man'ko, Phys. Rev. A, 49, 2993 (1994).

[42] V. V. Dodonov, O. V. Man'ko, and V. I. Man'ko, Phys. Rev. A, 50, 813 (1994).

[43] V. A. Andreev, V. I. Man'ko, O. V. Man'ko, and E. V. Shchukin, Theor. Math. Phys., 146, $172(2006)$.

[44] N. A. Ansari and V. I. Man'ko, Phys. Rev. A, 50, 1942 (1994).

[45] V. V. Dodonov, V. I. Man'ko, and D. E. Nikonov, Phys. Rev. A, 51, 3328 (1995).

[46] E. C. G. Sudarshan, Phys. Rev. Lett., 10, 277 (1963).

[47] R. J. Glauber, Phys. Rev., 131, 2766 (1963).

[48] R. F. Werner and M. M. Wolf, Phys. Rev. Lett., 86, 3658, (2001).

[49] V. V. Dodonov and V. I. Man'ko, Invariants and Evolution of Nonstationary Quantum Systems, in M. A. Markov (ed.), Proceedings of Lebedev Physics Insitute 183, Nova Science, Commack, NY (1989).

[50] C. F. Wildfeuer, A. P. Lund, and J. P. Dowling, Phys. Rev. A, 76, 052101 (2007).

[51] G. Schrade, V. M. Akulin, V. I. Man'ko, and W. P. Schleich, Phys. Rev. A, 48, 2398 (1993).

[52] A. A. Kolesnikov and V. I. Man'ko, J. Russ. Laser Res., 29, 142 (2008).

[53] B. S. Cirel'son, Lett. Math. Phys., 4, 93, (1980). 